Check for updates

Cite this: J. Mater. Chem. C, 2021, 9, 11216

\title{
Push-pull thiophene-based small molecules with donor and acceptor units of varying strength for photovoltaic application: beyond P3HT and PCBM†
}

\author{
Martina Marinelli, $\ddagger^{\mathrm{a}}$ Andrea Candini, (D) $\ddagger^{\mathrm{bc}}$ Filippo Monti, (D) $\ddagger^{\mathrm{b}}$ Alex Boschi, (D) ${ }^{\mathrm{b}}$ \\ Mattia Zangoli, (ID*bc Elisabetta Salatelli, (D) a Filippo Pierini, (D) d \\ Massimiliano Lanzi, (D) a Alberto Zanelli, (D) ${ }^{\mathrm{b}}$ Massimo Gazzano (D) ${ }^{\mathrm{b}}$ and \\ Francesca Di Maria (D) *bc
}

\begin{abstract}
Here is reported an expedient synthesis implementing enabling technologies of a family of thiophenebased heptamers alternating electron donor (D) and acceptor (A) units in a $D-A^{\prime}-D-A-D-A^{\prime}-D$ sequence. The nature of the peripheral A groups (benzothiadiazole vs. thienopyrrole-dione $v s$. thiophene-S,S-dioxide) and the strength of the donor units (alkyl vs. thioalkyl substituted thiophene ring) have been varied to finely tune the chemical-physical properties of the D-A oligomers, to affect the packing arrangement in the solid-state as well as to enhance the photovoltaic performances. The optoelectronic properties of all compounds have been studied by means of optical spectroscopy, electrochemistry, and density functional theory calculations. Electrochemical measurements and Kelvin probe force microscopy (KPFM) predicted a bifunctional behaviour for these oligomers, suggesting the possibility of using them as donor materials when blended with PCBM, and as acceptor materials when coupled with $\mathrm{P} 3 \mathrm{HT}$. Investigation of their photovoltaic properties confirmed this unusual characteristic, and it is shown that the performance can be tuned by the different substitution pattern. Furthermore, thanks to their ambivalent character, binary non-fullerene small-molecule organic solar cells with negligible values of HOMO and LUMO offsets were also fabricated, resulting in PCEs ranging between $2.54-3.96 \%$.
\end{abstract}

Received 8th June 2021, Accepted 23rd July 2021

DOI: $10.1039 / \mathrm{d} 1 \mathrm{tc} 02641 \mathrm{k}$

rsc.li/materials-c are driving the development of increasingly high-performing organic photovoltaic materials. ${ }^{4-7}$ Indeed, power conversion efficiencies (PCEs) of organic solar cells are now above $18 \%$, confirming the role of $\mathrm{BHJs}$ as a promising technology for future clean energy supply. ${ }^{8,9}$ In this context, great attention has been devoted to the design of small molecules, which if compared to their polymer counterparts present many advantages: (i) well-defined molecular structures, (ii) precise molecular weights, (iii) high purity without batch-to-batch variations (iv) solubility in non-chlorinated solvents. ${ }^{10-13}$ The current molecular design is based on the push-pull concept, in which electron donor (D) and electron acceptor (A) units are covalently linked and alternated along the conjugated backbone. ${ }^{14-16}$ The different nature of the D and A fragments allows modulating the shift of electron density from the $\mathrm{D}$ to the A moiety achieving a fine control of the optoelectronic properties such as a wider absorption range of the solar emission spectrum. ${ }^{17-22}$ Furthermore, well-engineered molecular moieties may help in guiding the morphology of the active layer thus eliciting suitable phase separation, one of the most demanding tasks to achieve high efficiency in 
$\mathrm{BHJ} .{ }^{23}$ Over the years, different push-pull small molecules, characterized by a wide diversity in the alternation of the $\mathrm{D}$ and $\mathrm{A}$ functional units, such as $\mathrm{D}-\mathrm{A}, \mathrm{D}-\mathrm{A}-\mathrm{A}^{\prime}, \mathrm{A}-\mathrm{D}-\mathrm{A}, \mathrm{D}-\mathrm{A}-\mathrm{D}$ sequence, have been synthesized to be employed as donors counterpart in blend with fullerene-based acceptors. ${ }^{24-28}$ Recently, researchers have also focused their attention on synthesizing small molecules capable of substituting fullerene derivatives to overcome the pitfalls related to their use - that include expensive synthesis, difficult tunability of the energy gap, and scarce absorption in the visible spectrum - thus allowing for the fabrication of non-fullerene small molecule organic solar cells (NF-SM OSCs). ${ }^{29,30}$ In this scenario, thiophene-based derivatives have a prominent role since they represent a unique class of compounds that have been proved to fulfill both the role of donor and acceptor materials or even that of single materials in $\mathrm{BHJ}$ solar cells. ${ }^{31-37}$ Interestingly, it has also been reported that some push-pull thiophene-based oligomers can display a bifunctional photovoltaic behavior, i.e., they can be employed both as a D material when blended with PCBM or as an A when combined with a D compound such as P3HT. ${ }^{38-40}$ This peculiar behaviour has been scarcely reported in the literature, as most photoactive materials exhibit only unipolar photovoltaic properties, i.e., they work either as donors or acceptors. Here, we report the synthesis and characterization of a novel class of $\mathrm{D}^{-} \mathrm{A}^{\prime}-$ D-A-D-A'-D co-oligomers that can be applied, depending on the active material partner, for the fabrication of different $\mathrm{BHJ}$ solar cells. Four oligomers (heptamers) with different $\mathrm{D}$ and peripheral A units were engineered. It was varied either the strength of the D unit by replacing alkylated thiophene units $\left(\mathbf{T} 7 \mathbf{B z}-\mathbf{B z} \mathbf{z}_{\mathbf{2}}\right)$ with thioalkylated thiophenes (T7SBz-Bz $\mathbf{z}_{2}$ ), or the peripheral A moieties by substituting the benzothiadiazole units with thienopyrrole-dione (T7Bz-Dp $\mathbf{p}_{\mathbf{2}}$ ) or thiophene $S, S$-dioxide moieties (T7Bz-TSO $\mathbf{S}_{2}$ ) (Chart 1).

The effects of the different substitution patterns (i.e., the presence of additional sulfur atoms attached to alkyl chains or the combination of different acceptor units) on the electronic properties in the solid state, as well as on their packing and nanoscale blend morphologies were investigated. Different characterization techniques, including X-ray diffraction (XRD), differential scanning calorimetry (DSC), UV-vis spectroscopy, cyclic voltammetry (CV), Kelvin probe force microscopy (KPFM) and atomic force microscopy (AFM) were employed for this purpose. Furthermore, density functional theory (DFT) calculations enabled to gain a deeper understanding concerning the electronic properties of the investigated materials at the molecular level.

We show that this family of heptamers presents multiple capabilities of working as active materials in $\mathrm{BHJ}$ solar cells either as donor material in blend with PCBM or as acceptor in blend with P3HT. Furthermore, we report that couples of oligomers with small HOMO $\left[\Delta E(\mathrm{HOMO})_{\text {offset }}-0.01\right.$ to $\left.0.00 \mathrm{eV}\right]$ and LUMO offsets [ $\left.\triangle E(\text { LUMO })_{\text {offset: }} 0.07-0.11 \mathrm{eV}\right]$ can be employed for the realization of binary NF-SM OSCs, ${ }^{41-43}$ using non-chlorinated solvent for their deposition. Finally, the environmentally friendly approach employed for the deposition of the oligomeric blends allows achieving higher $\mathrm{BHJ}$ performance when compared to cells deposited from chlorinated solvent.

\section{Results and discussion}

\section{Synthesis and characterization of heptamers}

Scheme 1 shows the synthetic pattern employed for the preparation of push-pull heptamers characterized by a D-A sequence. Benzothiadiazole (Bz), thienopyrrole-dione (Dp) or thiophene $S, S$-dioxide (TSO) moieties were employed as A unit, while hexyl (Hex) or thiohexyl (SHex) substituted thiophene ring as D unit. As depicted in Scheme 1, all synthetic steps, except for the lithiation of compound 2, take advantage of enabling technologies. In particular, ultrasound (US) assistance for bromination reactions and microwave (MW) irradiation for Suzuki and Stille cross-coupling reactions. In agreement with what was reported in previous works, these techniques allow to (i) radically improve reaction yields, (ii) lower reaction times

\section{$D-A^{\prime}-D-A-D-A^{\prime}-D$ heptamers}
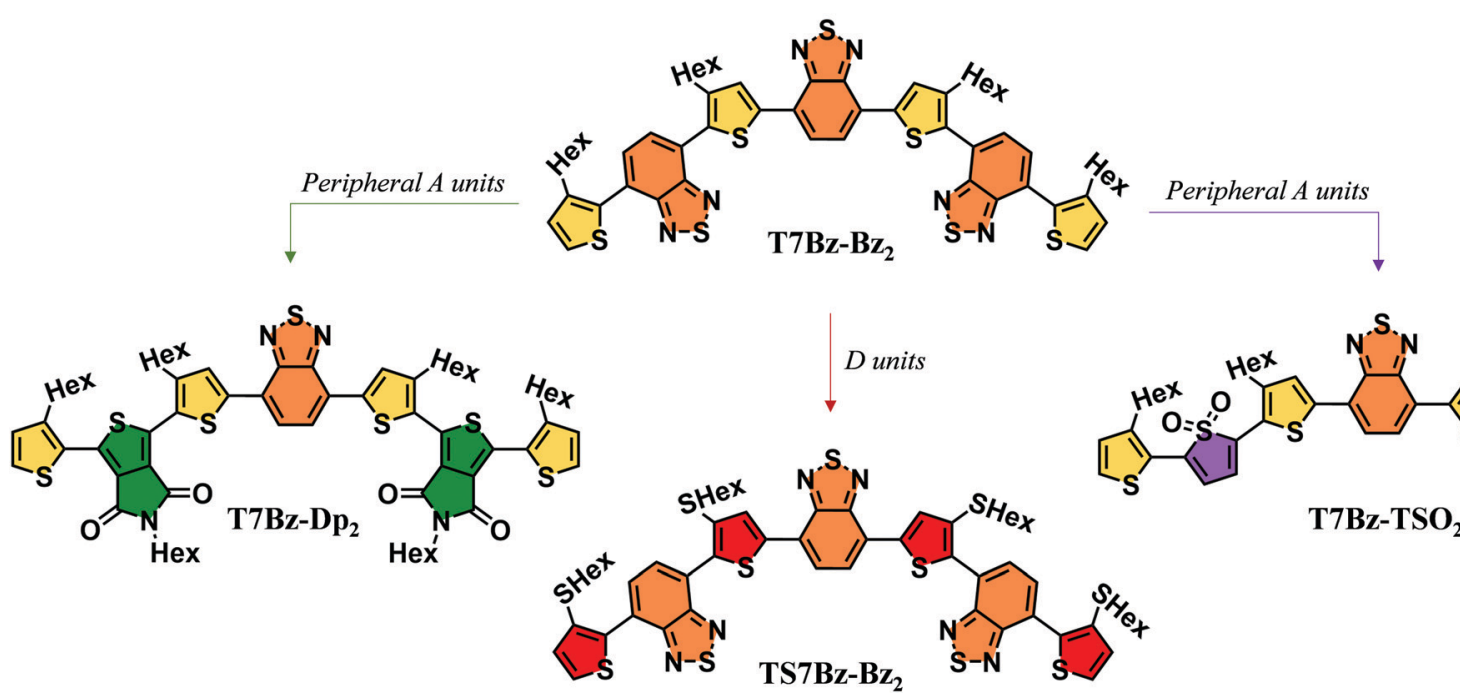

Chart 1 Molecular structures of the oligomers. 


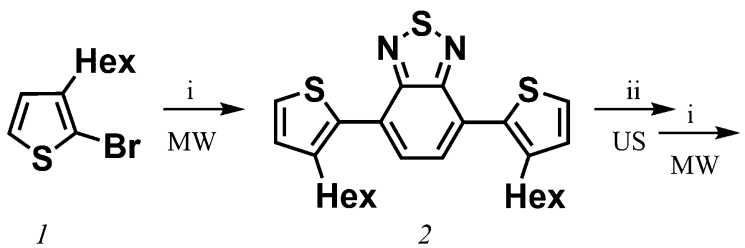

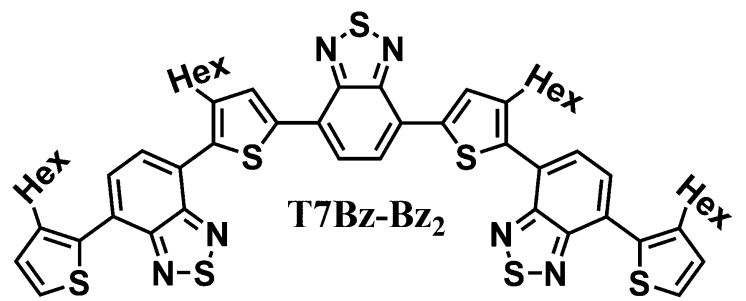<smiles>CC1CC1</smiles><smiles>[Y]Oc1ccsc1-c1sc(-c2sccc2[R4])c2c1C(=O)N([R10])C2=O</smiles>

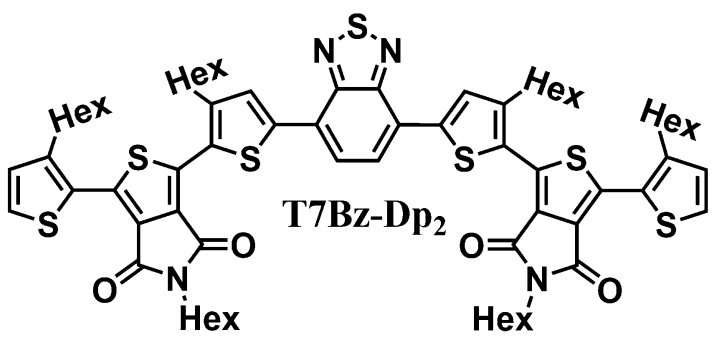

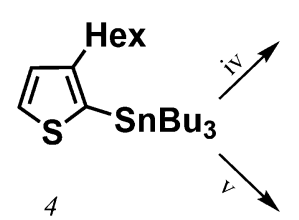

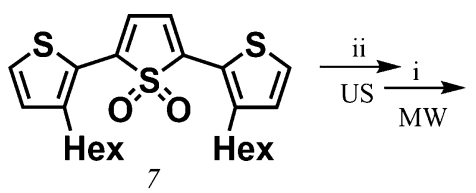

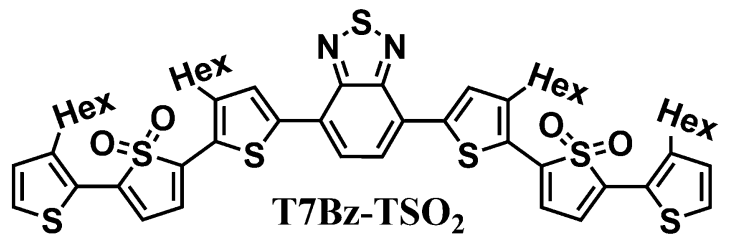

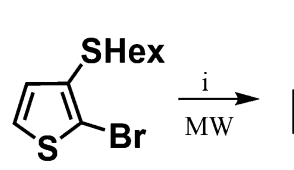

$1 s$

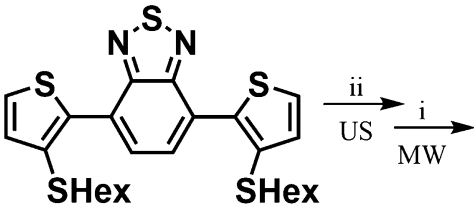

$2 s$

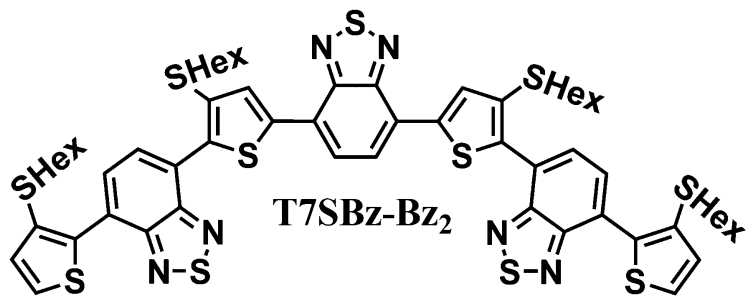

Scheme 1 Synthetic pattern for the synthesis of heptamers. (i) 4,7-Bis(4,4,5,5-tetramethyl-1,3,2-dioxaborolan-2-yl)benzo[c][1,2,5]thiadiazole, NaHCO 3 , $\mathrm{Pd}(\mathrm{dppf}) \mathrm{Cl}_{2}, \mathrm{THF} / \mathrm{H}_{2} \mathrm{O}(1 / 2), 80{ }^{\circ} \mathrm{C}$; (ii) $\mathrm{N}$-bromosuccinimide, $\mathrm{CH}_{2} \mathrm{Cl}_{2}, 25{ }^{\circ} \mathrm{C}$; (iii) $n \mathrm{BuLi}, \mathrm{Sn}(\mathrm{Bu}){ }_{3} \mathrm{Cl}$, $\mathrm{THF}_{\text {(dry), }}-78{ }^{\circ} \mathrm{C}$; (iv) $1,3-$ dibromo-5-hexyl-4Hthieno[3,4-c]pyrrole-4,6(5H)-dione, $\mathrm{Pd}\left(\mathrm{PPh}_{3}\right)_{4}$, toluene, $110{ }^{\circ} \mathrm{C}$; (v) 2,5-dibromothiophene 1,1-dioxide, $\mathrm{Pd}\left(\mathrm{PPh}_{3}\right)_{4}$, toluene, $110{ }^{\circ} \mathrm{C}$.

from hours to minutes, and (iii) work in environment-friendly conditions using water as co-solvent. ${ }^{44-46}$ In the final step, all heptamers, which differ for the peripheral A units or for the presence of an extra sulfur atom in the side chains of the $\mathrm{D}$ group, were obtained in yield ranging from 60 to $70 \%$ after 20 min of MW irradiation. The presence of hexyl side chains on the four thiophene rings guarantees good solubility of all molecules in DCM, toluene, and THF, particularly for T7Bz-Dp $\mathbf{p}_{2}$, which has additional hexyl side chains installed on the Dp units. The details of the synthesis are reported in ESI. $\dagger$

DSC analysis was performed to obtain information on the effects of the substitution pattern on the materials' thermal characteristics and to provide guidelines for device optimization. Table S1 (ESI $\dagger$ ) summarizes the transition temperatures and their enthalpy values. DSC analysis revealed that the thermal properties of the heptamers are deeply influenced by the nature of the $\mathrm{D}$ and $\mathrm{A}$ units present in the molecular structure (Fig. 1A and B).
$\mathbf{T} 7 \mathbf{B z}-\mathbf{B z}_{2}$ exhibit only a glass transition (Tg) suggesting its persistence in an amorphous state over all the investigated temperature range. Remarkably, the introduction of sulfur atoms in the side chains of the D moieties $\left(\mathbf{T} 7 \mathbf{S B z}-\mathbf{B z}_{2}\right)$ or the

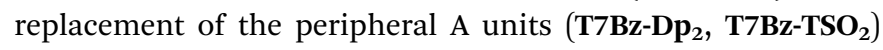
results in DSC plots characterized by one or two endothermic transitions. In particular, the presence of TSO groups as peripheral A units shifts the endothermic processes to a higher temperature (Table S1, ESI $\dagger$ ). Note that, for all compounds, the observed transitions occur far below their thermal decompositions which are higher than $250{ }^{\circ} \mathrm{C}$ (see TGA analysis in ESI $\dagger$ ). To better evaluate the nature of the different phases, XRD analysis was performed. The diffraction profiles of the films of the investigated materials are reported in Fig. 1C. The room temperature XRD pattern of $\mathbf{T} 7 \mathbf{B z}-\mathbf{B z} \mathbf{z}_{2}$ confirms its amorphous nature since no sharp peaks are observed. In $\mathbf{T} 7 \mathbf{S B z}-\mathbf{B z} \mathbf{z}_{2}$, the presence of sulfur atoms in the alkyl chains of the thiophene units induces a partial order, indeed the diffraction profile 

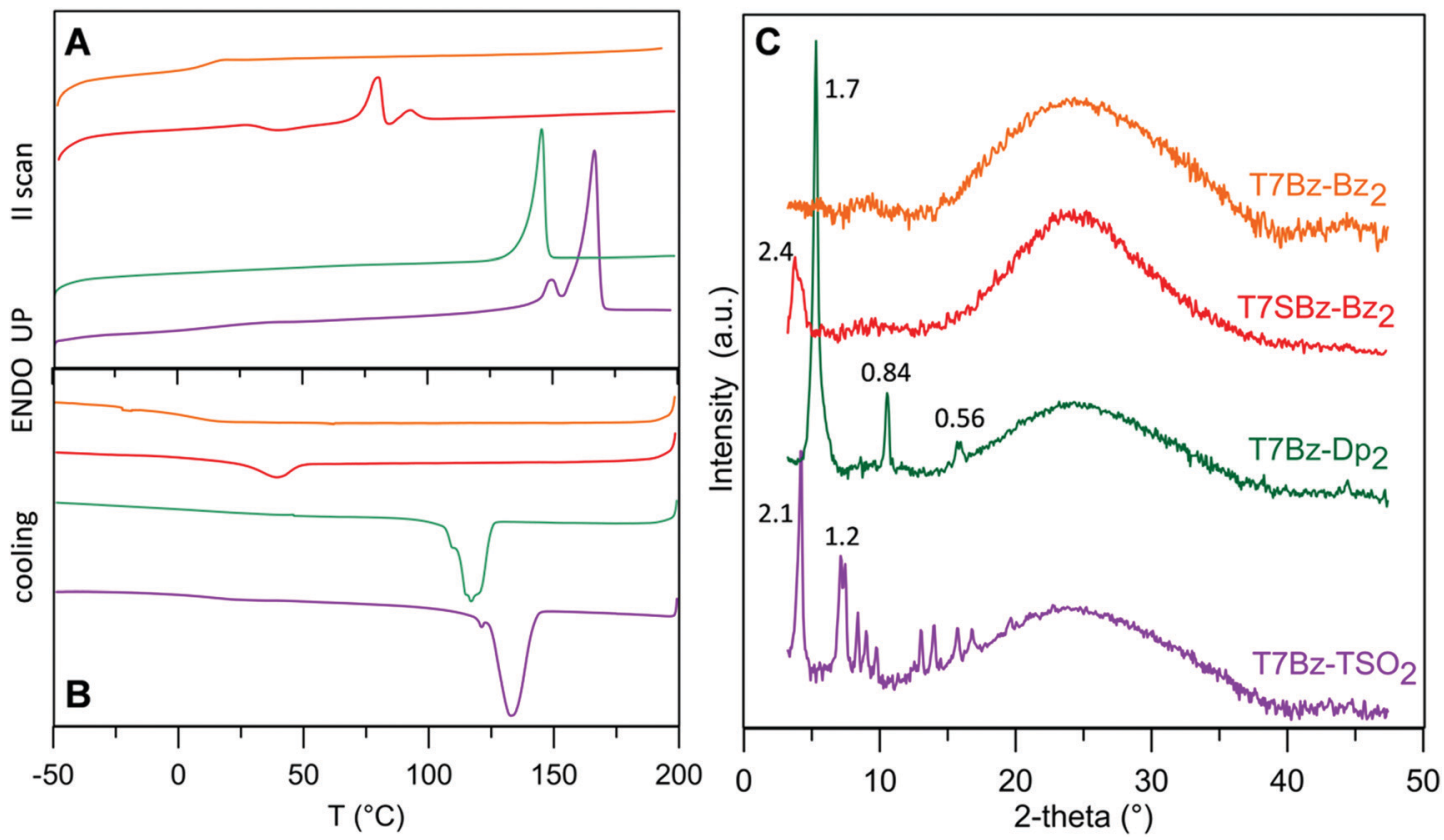

Fig. 1 (A) DSC: second heating and (B) cooling scans, at $10{ }^{\circ} \mathrm{C} \mathrm{min}^{-1}$. (C) XRD: scans of heptamers films on glass substrates (orange line $\mathrm{T7Bz}-\mathrm{Bz} z_{2}$; red line $\mathbf{T 7 S B z}-\mathbf{B z}_{2}$; green line $\mathbf{T 7 B z}-\mathrm{Dp}_{2}$; purple line $\mathbf{T 7 B z}-\mathrm{TSO}_{2}$ ). $Y$-Axis is plotted as Log scale because of the great difference in peak intensity; taking this into account it causes expansion of the bell-shaped background due to glass support. Interlayer distances are reported in nm. Arbitrary vertical shifts are applied in both $\mathrm{A}$ and $\mathrm{B}$ panels to minimize overlaps.

shows only one peak $(2.4 \mathrm{~nm})$ as usual for mesomorphic materials. ${ }^{47}$ Substitution of the peripheral A units of $\mathbf{T} 7 \mathbf{B z}-\mathbf{B z}_{\mathbf{2}}$ with Dp or TSO appears to induce a higher propensity to form ordered arrays. Indeed, the pattern obtained after replacing peripheral $\mathrm{Bz}$ units with $\mathrm{Dp}$ shows only three peaks at $5.3^{\circ}$, $10.5^{\circ}, 15.8^{\circ}$, all belonging to a periodicity of $1.7 \mathrm{~nm}$, suggesting the presence of a highly oriented film or a layer ordered smectic system. On the other hand, exchanging the $\mathrm{Bz}$ units with TSO brings to a fully tridimensional array, indeed, the XRD T7 Bz-TSO ${ }_{2}$ pattern is characteristic of a crystalline material.

The combination of calorimetric and XRD data allows a better insight into the solid-state morphology of the materials. With respect to $\mathbf{T} \mathbf{7} \mathbf{B z}-\mathbf{B z}_{2}$, the $\mathbf{T} 7 \mathbf{S B z}-\mathbf{B z}_{2}$ sample presents multiple endothermic processes, with the first endotherm peak at $81{ }^{\circ} \mathrm{C}\left(\Delta H=14.24 \mathrm{~J} \mathrm{~g}^{-1}\right)$ bigger than the second one at $93{ }^{\circ} \mathrm{C}$ $\left(\Delta H=4.01 \mathrm{~J} \mathrm{~g}^{-1}\right)$ (Table S1, ESI $\left.\dagger\right)$. Since XRD reveals only one peak roughly correspondent to the molecular length, absent in $\mathbf{T} 7 \mathbf{B z}-\mathbf{B z}_{2}$, and DFT calculation suggest that the molecule presents a bent geometry resembling that of a banana (see below) well known for promoting the organization in liquid crystalline phases $^{48}$ - a transformation between mesomorphic phases before melting can be hypothesized. This result highlights the importance of the extra sulfur atom in the side chains, which through S $\cdots \mathrm{S}$ non-covalent intermolecular interactions favors an extra long-range ordering in $\mathbf{T} 7 \mathbf{S B z}-\mathbf{B z}_{2} \cdot{ }^{45,46,49}$ Replacing the peripheral $\mathrm{Bz}$ unit with $\mathrm{Dp}$ and TSO induces an increase in the crystalline character of the compounds. The Dp units in T7Bz-Dp $\mathbf{p}_{2}$ promote a long-range (layer) order, as evidenced by the appearance of an endothermic peak at $146{ }^{\circ} \mathrm{C}$ relatives to the melting of the material, in a fully reversible process, as confirmed by the exothermic peak in cooling scan corresponding to its crystallization. The presence of Dp probably enforces backbone co-planarity via intermolecular $\mathrm{S} \cdots \mathrm{O}$ interactions leading to close $\pi-\pi$ stacking in the solid-state. ${ }^{50} \mathrm{In}$ compound $\mathbf{T} 7 \mathbf{B z}-\mathbf{T S O}_{2}$, the TSO units determine a double endothermic transition that can be ascribed to melt/recrystallization processes involving defective crystals during the DSC scan, or to the existence of a high-temperature phase, which is not markedly different from the crystalline phase present at room temperature since the heat exchange is quite small $\left(\Delta H_{\mathrm{m}}=2.95 \mathrm{~J} \mathrm{~g}^{-1}\right)$.

\section{DFT electronic structure calculations}

DFT calculations reveal that all the heptamers have a large number of conformers that can be populated at room temperature. To get a deeper understanding of such structural freedom, a conformational analysis has been carried out on trimers constituting the investigated heptamers (Scheme S5 and Fig. $\mathrm{S} 20, \mathrm{ESI} \dagger)$. Although this analysis merely takes into account the conformational freedom for the isolated molecule without considering any intramolecular interactions, such as $\pi-\pi$ interactions, chain interdigitations, etc., it can qualitatively elucidate experimental finding. For instance, the presence of sulfur atoms in the alkyl chains nearby the $\mathrm{Bz}$ units (as in $2 \mathbf{s}$, the precursor of $\mathbf{T} 7 \mathbf{S B z}-\mathbf{B z}_{2}$ ) strongly destabilizes the anti conformations, in which the sulfur electron density is forced to interact with the nitrogen lone pairs of the Bz unit (i.e., syn/anti ratio of $72 / 28$ at $298 \mathrm{~K}$, see Table S2, ESI $\dagger$ ). On the contrary, the absence of sulfur-functionalized side chains (as in 2, the precursor of $\mathbf{T} 7 \mathbf{B z}-\mathbf{B z}_{2}$ ) strongly reduces this effect and the syn and anti 
conformers are nearly isoenergetic, leading to the presence of a plethora of thermally accessible conformers at room temperature and, most probably, explaining the amorphous nature of $\mathbf{T} 7 \mathbf{B z}-\mathbf{B z}_{2}$ in contrast to the partial order found in $\mathbf{T} 7 \mathbf{S B z}-\mathbf{B z} \mathbf{z}_{2}$ (see the previous section). An even more pronounced effect is observed when the $\mathrm{Bz}$ unit is replaced by TSO (as in 7, the precursor of $\mathbf{T} 7 \mathbf{B z}-\mathbf{T S O}_{2}$ ). Indeed, the strong intramolecular S. . O interactions between the sulfonyl group of the TSO unit and the neighboring thiophene rings are able to strongly stabilize the syn conformers (i.e., syn/anti ratio of 75/25 at $298 \mathrm{~K}$ ); notably, this stabilization is maximized in $C_{2}$ symmetry which favors the formation of two S . . O interactions, instead of a single $\mathrm{S} \cdots \mathrm{O} \cdots \mathrm{S}$ one as in the $C_{\mathrm{s}}$ group (see Table S2, ESI $\dagger$ ). ${ }^{51}$ The presence of a well-defined minimum conformation can indeed be a reason for the higher degree of crystallinity observed experimentally in T7Bz-TSO $\mathbf{T}_{2}$ (see DSC and XRD characterizations). Differently, the introduction of the Dp unit does not induce any significant intramolecular stabilization despite its high coplanarity and the presence of carbonyl and amide groups.

Fig. 2 shows the frontier molecular orbitals of the lowestenergy conformer of each of the investigated heptamers. For all molecules, the HOMO is mainly located on the central D-A-D unit, which is more or less stabilized depending on the different peripheral A units. In particular, the presence of the electron-donating sulfur atoms in the alkyl chains of T7SBz- $-\mathbf{B z}_{2}$ induces a slight HOMO destabilization, if compared to the pristine $\mathbf{T} 7 \mathbf{B z}-\mathbf{B z} \mathbf{z}_{2}$ heptamer (i.e., $+0.08 \mathrm{eV}$ ). On the contrary, the presence of the strongly electron-accepting peripheral units in $\mathbf{T} 7 \mathbf{B z}-\mathbf{D p}_{\mathbf{2}}$ and $\mathbf{T} \mathbf{7} \mathbf{B z}-\mathbf{T S O}_{\mathbf{2}}$ is capable of inducing a HOMO stabilization of 0.16 and $0.15 \mathrm{eV}$, respectively.

The LUMO of all heptamers is predominantly located on the lowest $\pi^{*}$ molecular orbital of the central Bz moiety, with some degree of delocalization to the nearby units. As in the case of HOMO, the LUMO energy can be tuned along with the series roughly depending on the acceptor strength of the peripheral moieties (i.e., $\mathbf{T} 7 \mathbf{B z}-\mathbf{T S O} \mathbf{2}_{2}<\mathbf{T} 7 \mathbf{B z}-\mathbf{D p}_{2} \approx \mathbf{T} 7 \mathbf{S B z}-\mathbf{B z} \mathbf{z}_{2}<\mathbf{T} 7 \mathbf{B z}-\mathbf{B z}_{2}$ ).
It is worth noting that, for all the investigated molecules, while the LUMO is localized on the central acceptor moiety, the LUMO+1 and the LUMO+2 are predominantly located on the peripheral acceptors, with the latter generally having a greater delocalization on the central Bz as well (see Fig. 2).

\section{Electro-optical characterizations}

Fig. S23 (ESI $\dagger$ ) shows the cyclovoltammetry of the heptamers in solution, and thin film and Table 1 reports their redox potentials, HOMO and LUMO energy levels, and energy gaps.

As shown in Table 1, increasing the strength of the donor units (Hex vs. SHex), results in a significant shift in the oxidation and reduction potentials both in solution and thin film. Indeed, compared to $\mathbf{T} \mathbf{7} \mathbf{B z}-\mathbf{B z}_{2}, \mathbf{T} \mathbf{T} \mathbf{S B z}-\mathbf{B z}_{2}$ exhibits an oxidation wave shifted towards less positive potentials (180 $\mathrm{mV}$ both in solution and in thin film) and the reduction ones towards less negative potentials $(60 \mathrm{mV}$ in solution and $70 \mathrm{mV}$ in thin film). These results are in good agreement with the HOMO destabilization and the LUMO stabilization estimated by DFT calculations on $\mathbf{T} 7 \mathbf{S B z}-\mathbf{B z}_{2}$, if compared to pristine $\mathbf{T} 7 \mathbf{B z}-\mathbf{B z}_{\mathbf{2}}$ (see above). Furthermore, in thin films, the two oligomers show the same redox potentials of the molecules in solution, indicating that neither conformation changes nor orbital staking occurs. This result agrees with both XRD measurements, as these heptamers provide amorphous or low crystalline films, and absorption spectra. Replacing the peripheral acceptor units of $\mathrm{Bz}$ with Dp or TSO affects redox potentials depending on the strength of the A units and the packing arrangement. $^{52}$ In solution, both $\mathbf{T} \mathbf{7} \mathbf{B z}-\mathbf{D p}_{\mathbf{2}}$ and $\mathbf{T} \mathbf{7} \mathbf{B z}-\mathbf{T S O}_{2}$ result to be more difficult to oxidize and easier to reduce than $\mathbf{T} 7 \mathbf{B z}-\mathbf{B z} z_{2}$. In detail, $\mathbf{T} 7 \mathbf{B z}-\mathbf{D p}_{2}$ shows oxidation and reduction waves shifted by 70 and $100 \mathrm{mV}$, respectively. Similarly, T7Bz-TSO ${ }_{2}$ shows a shifted oxidation wave of $60 \mathrm{mV}$ at more positive potentials and a reduction wave that is remarkably shifted by $220 \mathrm{mV}$ to a less negative potential. Such experimental findings match well with the scenario already depicted by DFT calculation, considering dichloromethane solvation effects. Conversely, in

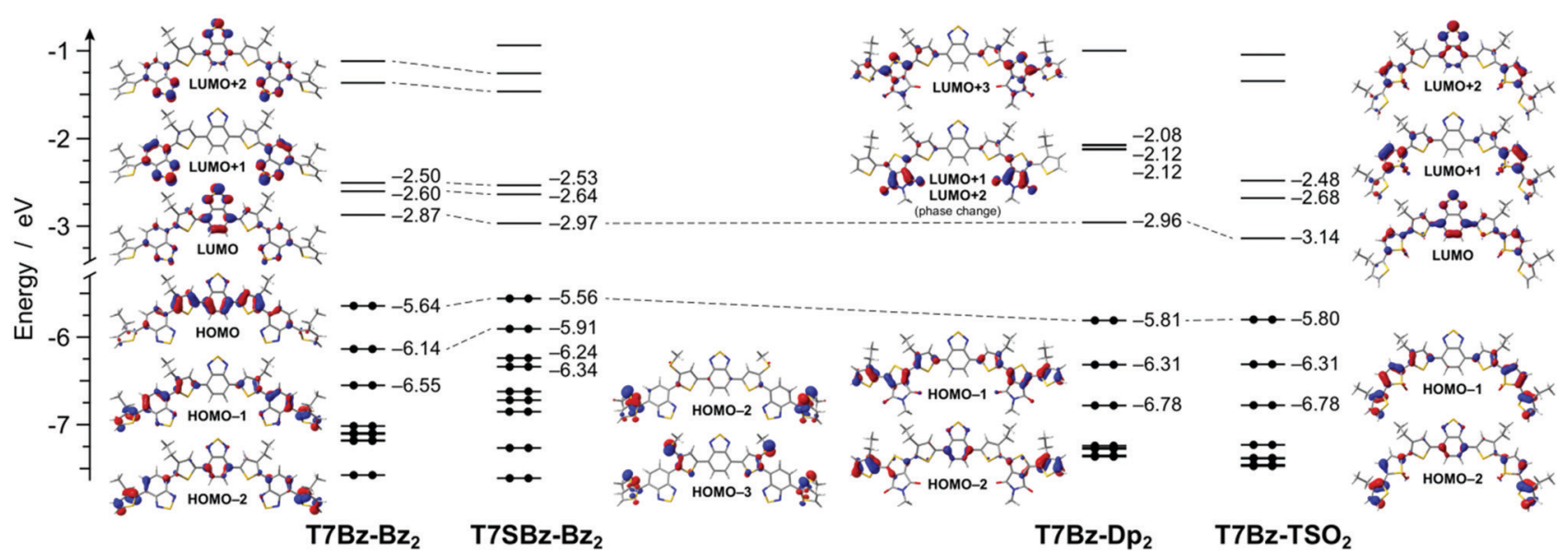

Fig. 2 Energy diagram showing the energy values of the frontier Kohn-Sham molecular orbitals of the investigated heptamers in dichloromethane. For some relevant orbitals, the corresponding isosurface is also displayed (isovalue $=0.04 \mathrm{e}^{1 / 2} \mathrm{bohr}^{-3 / 2}$ ). Along with the series, relevant orbitals with similar topology are connected with a dashed line for easier comparison. 
Table 1 Redox potentials and absorptions of heptamers in solution and thin film

\begin{tabular}{|c|c|c|c|c|c|c|c|c|c|}
\hline & $E_{\mathrm{Ox}}{ }^{a}$ & $E_{\text {red }}^{a}$ & $E_{\mathrm{ox}}^{b}$ & $E_{\text {red }}^{b}$ & HOMO $^{b}$ & LUMO $^{b}$ & $E_{\mathrm{g}}{ }^{b}$ & $\lambda_{\mathrm{abs}}{ }^{a}$ & $\lambda_{\mathrm{abs}}{ }^{b}$ \\
\hline T7Bz-Dp 2 & +1.17 & -1.08 & +0.90 & -1.23 & -5.58 & -3.45 & 2.13 & 380,498 & 380,494 \\
\hline $\mathrm{T} \mathrm{Bz}-\mathrm{TSO}_{2}$ & +1.16 & -0.96 & +0.89 & -1.05 & -5.57 & -3.63 & 1.94 & 316,522 & 315,533 \\
\hline
\end{tabular}

${ }^{a}$ Solution. ${ }^{b}$ Thin film ( $E_{\mathrm{ox}}$ and $E_{\text {rid }}$ in V; HOMO, LUMO and $E_{\mathrm{g}}$ in $\mathrm{eV} ; \lambda$ in $\mathrm{nm}$ ).

thin film, their redox potentials strongly differ from the solution because of the molecular packing. Indeed, $\mathbf{T} 7 \mathbf{B z}-\mathbf{T S O}_{\mathbf{2}}$ and $\mathbf{T} \mathbf{7} \mathbf{B z}-$ $\mathbf{D p}_{2}$, which after deposition in thin film give crystalline films, show less positive oxidation potentials and less negative reduction potentials than in solution. This induces a consequent shortening of the $E_{\mathrm{g}}$ as a result of a $\pi-\pi^{*}$ staking in the crystals (Table 1 ). Notably, a comparison of the HOMO/LUMO energy levels of the heptamers with those of P3HT and PCBM highlights that LUMO levels of these compounds lie in an energetic position whereby electron transfer can occur either from P3HT or to PCBM (Fig. S24, ESI $\dagger$ ). The absorption spectra reflect the trend of the $E_{\text {g }}$ determined by CV measurements. All compounds, both in solution and thin film, exhibit the typical behaviour of alternating D-A conjugated molecules with a two-band absorption profile in the UV-visible region relative to internal charge-transfer (ICT) and $\pi \rightarrow \pi^{*}$ transition processes. ${ }^{53}$ As shown in Fig. 3, ICT absorption bands are susceptible to variations in the D or A groups. Indeed, the ICT maxima shift to higher wavelengths when different A units are present (TSO-Bz $>\mathrm{Dp}-\mathrm{Bz}>\mathrm{Bz}-\mathrm{Bz}$ ) or by increasing the electron-donating effect of the donor groups (SHex $>$ Hex) (Table 1). The heptamers exhibit similar molar extinction coefficients $(\varepsilon)$ of $\sim 3.7 \times 10^{4} \mathrm{M}^{-1} \mathrm{~cm}^{-1}$ (Fig. 3A and Table S8, ESI $\dagger$ ).

TD-DFT calculations, carried out at the same level of theory used for ground-state optimizations, are able to qualitatively reproduce the absorption profiles of all heptamers, despite a general underestimation of around $0.4 \mathrm{eV}$, if vertical transition energies are directly compared to experimental absorption maxima (Fig. S21a, ESI $\dagger$ ). This phenomenon can be attributed
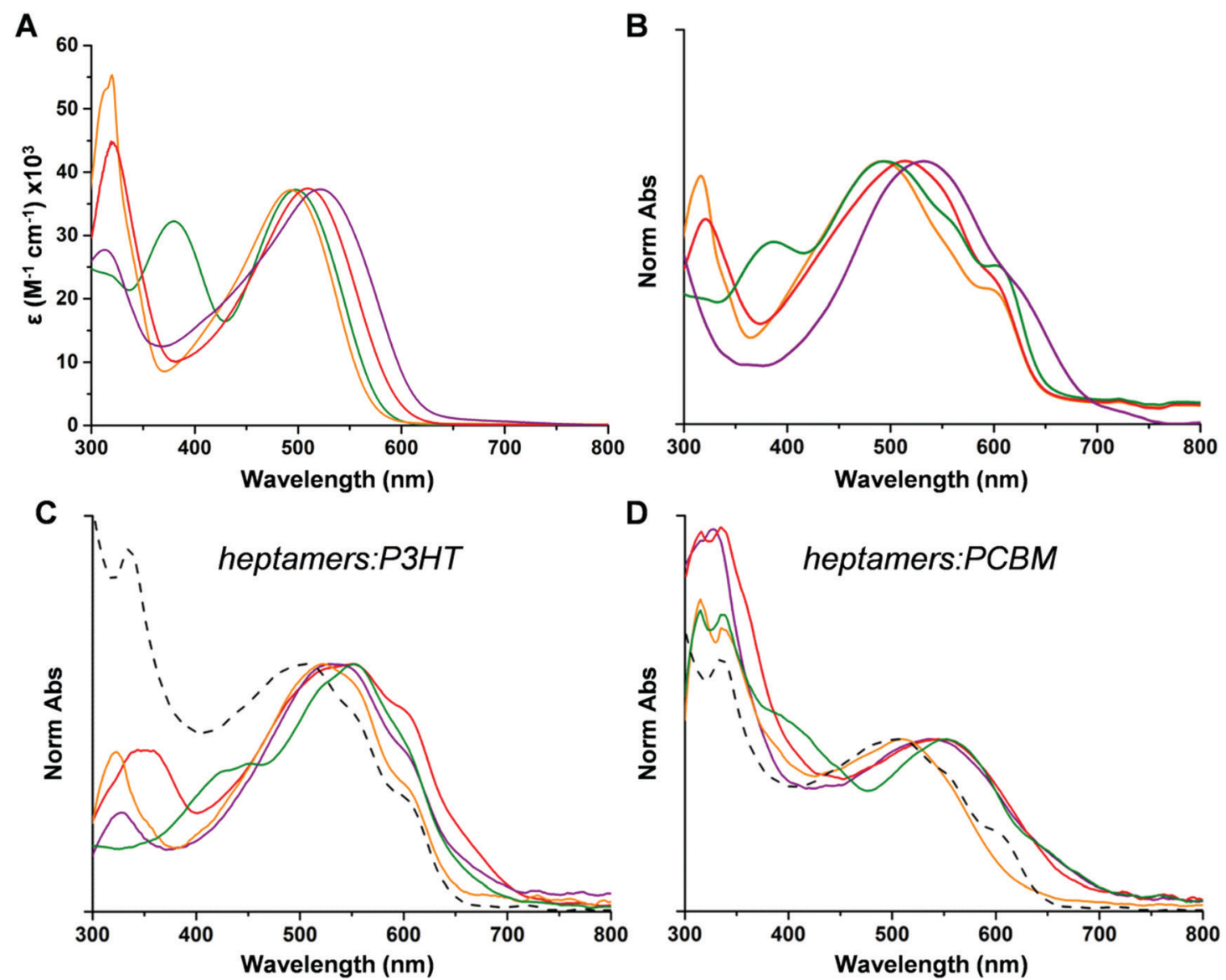

Fig. 3 (A and B) Absorption spectra of heptamers in $\mathrm{CH}_{2} \mathrm{Cl}_{2}$ solution and thin film, respectively; (orange line $\mathbf{T 7} \mathbf{B z}-\mathbf{B z}_{\mathbf{2}}$; red line $\mathbf{T 7 S B z}-\mathbf{B z} \mathbf{z}_{\mathbf{2}}$; green line T7Bz-Dp $\mathbf{p}_{2} ;$ purple line T7Bz-TSO${ }_{2}$ ). (C and D) Thin film absorption spectra of heptamers in blend with P3HT and PCBM (1:1 w/w). For comparison, the thin film spectra of P3HT: PCBM blend $(1: 1 \mathrm{w} / \mathrm{w})$ is reported as dashed black line. 
to the important charge-transfer contribution associated with such transitions, which can be better taken into account by increasing the amount of Hartree-Fock exchange in the functional. ${ }^{54}$ Indeed, when M06-2X is used, the agreement with experimental data is excellent (Fig. S21b, ESI $\dagger$ ); anyway, only minor differences have been observed in the nature of the transitions calculated either by M06 or M06-2X functional, the latter leading only to more multiconfigurational excitations. For all the heptamers, the intense and unstructured absorption band in the region between 450 and $600 \mathrm{~nm}$ is almost exclusively attributed to a nearly pure HOMO $\rightarrow$ LUMO excitation, leading to the population of $\mathrm{S}_{1}$ (Tables S3-S6, ESI $\dagger$ ). In the case of $\mathbf{T} 7 \mathbf{B z}-\mathbf{T S O}_{2}$, a distinct shoulder is experimentally observed next to $\mathrm{S}_{0} \rightarrow \mathrm{S}_{1}$ absorption band, at approx. $450 \mathrm{~nm}$. This transition is due to the $\mathrm{S}_{0} \rightarrow \mathrm{S}_{2}$ excitation, attributed to a charge transfer from the thiophene donors to the lateral TSO acceptors (Table S6, ESI $\dagger$ ). It is worth mentioning that TD-DFT calculations were also able to predict the presence of the second broad and unstructured absorption band around 350-440 nm, which is present only in the spectrum of T7Bz$\mathbf{D p}_{2}$. This band is attributed to an envelope of four vertical excitations (i.e., from $\mathrm{S}_{0}$ to $\mathrm{S}_{2}-\mathrm{S}_{5}$ ) that are basically CT transitions from the lateral thiophene units to either the 5-membered thiadiazole ring $\left(\mathrm{S}_{2}\right.$ and $\left.\mathrm{S}_{3}\right)$ or the Dp ones $\left(\mathrm{S}_{4}\right.$ and $\mathrm{S}_{5}$, Table $\mathrm{S} 5$, ESI $\dagger$ ). As reported in Fig. S25 (ESI $\dagger$ ), the heptamers exhibit a broad fluorescence band in the region between $550 \mathrm{~nm}$ and $750 \mathrm{~nm}$ with quantum yield in the range of $\sim 1-38 \%$ (see details in ESI $\dagger$ ). In thin film, the maximum absorption spectra of all heptamers remain approximately the same as in solution (Fig. 3B); additionally, band broadening and vibronic structuration suggest that solid-state organization promotes a better packing within molecules, particularly for $\mathbf{T} 7 \mathbf{B z}-\mathbf{D} \mathbf{p}_{2}$ and $\mathbf{T} 7 \mathbf{B z}$ $\mathbf{T S O}_{2}$. Indeed, TD-DFT calculations indicate a general flattening of all heptamers upon excited-state relaxation on S1 (Fig. S22 and Table S7, ESI $\dagger$ ). When blended with either P3HT or PCBM, the maxima of the thin film absorption spectra remain approximately the same and result to be red-shifted compared to the P3HT:PCBM blend, unveiling an enhancement of visible spectral coverage when using the heptamer (Fig. 3C and D). Analogously to P3HT:PCBM blend, the emission is significantly quenched, thus indicating that effective photoinduced charge transfer occurs between the heptamer and PCBM or P3HT (Fig. S27, ESI $\dagger$ ).

\section{KPFM measurements}

The possibility of using these heptamers as both $\mathrm{D}$ and $\mathrm{A}$ materials was tested by KPFM in two model organic solar cells systems, made of ITO/PEDOT:PSS/on top of which a thick layer of $\mathbf{T} 7 \mathbf{S B z}-\mathbf{B z}_{2}$ blended with P3HT or PCBM, respectively, was deposited (see ESI $\dagger$ ). KPFM allows imaging the surface potential (SP) distribution of the system under investigation (that is the difference between the tip and the sample work function (WF): $\mathrm{SP}=\mathrm{WF}_{\text {tip }}-\mathrm{WF}_{\text {sample }}$ ), simultaneously with the topographic information acquired in the conventional AFM mode. ${ }^{5,56}$ By investigating the sample before and after illumination, the measured changes of the SP values are indicative of the charge distribution induced by light excitation and whether and which carriers are exchanged with the substrate. Fig. 4A shows the topography and corresponding SP (under dark and illumination conditions) images of $\mathbf{T} 7 \mathbf{S B z}-\mathbf{B z} \mathbf{z}_{2}$ blended with P3HT (upper panels) and with PCBM (lower panels). The blend with $\mathrm{P} 3 \mathrm{HT}$ exhibits an amorphous and rough morphology with the presence of clusters with a size of tens of nanometers (Fig. $4 \mathrm{~A}(\mathrm{a})$ ). The clusters have a higher SP value (Fig. $4 \mathrm{~A}(\mathrm{~b})$ ) and hence a lower WF, thus they are associated with P3HT crystal domains. Similarly, the T7SBz-Bz $\mathbf{B z}_{2}$ compound is amorphous also in the blend with PCBM (Fig. 4A(d)), which forms round-shaped regions of approximately 200-300 $\mathrm{nm}$ of diameter, presenting the expected lower value of SP as shown in Fig. $4 \mathrm{~A}(\mathrm{e})$. When the systems are measured under illumination with a halogen lamp (Fig. 4A(c, f)), an evident shift of the average SP values for each blend is observed; interestingly, the changes have an opposite sign, i.e., the SP upon illumination decreases in the case of the P3HT blend, while it increases when the oligomer is mixed with PCBM.

This observation can be understood by considering the HOMO/LUMO energy levels alignment between the materials (see Fig. 4B, inset): for each blend, the charge exchange between the photoactive material and the substrate is favored only for one type of carriers and hindered for the others. When the P3HT (PCBM) is present, electrons (holes) are exchanged towards the PEDOT:PSS/ITO electrode, leaving the blend with an excess of holes (electrons) and hence a negative (positive) surface photovoltage SPV (i.e., the SP difference between the light and dark condition) is measured. This picture is schematized in Fig. 4B, where the histogram distribution of the SPV values is shown for the two blends, along with the corresponding HOMO/LUMO energy levels alignments. These measurements confirm the bifunctional nature of the heptamers and the possibility of using them as both D and A materials. Finally, the absence of clear charge separation between the materials of the blends may be ascribed to the fact that a thick film is being investigated, in agreement with previous reports on other organic photovoltaic cells. ${ }^{57}$

\section{Photovoltaic properties}

To evaluate the bifunctional photoactive behaviour of the heptamers, air-manufactured $\mathrm{BHJ}$ organic solar cells with a ITO/PEDOT:PSS/active layer/Al configuration were fabricated. The active layers were constituted of a $1: 1$ weight ratio blend of the heptamers with either PCBM or P3HT employed as A and D derivatives, respectively. The photovoltaic performance parameters for the prepared devices are listed in Table 2, while the corresponding $J-V$ features of the best-performing solar cells and external quantum efficiency (EQE) curves are reported in Fig. 5.

Heptamers:PCBM blends. Heptamers, when utilized as donor materials in blend with PCBM, provide PCE values in the range of $1.60-4.32 \%$. As shown in Table 2, replacing the peripheral acceptor units of $\mathbf{T} \mathbf{7} \mathbf{B z}-\mathbf{B} \mathbf{z}_{\mathbf{2}}$ induces a small decrease in the photoconversion efficiency - going from $1.91 \%$ to $1.83 \%$ for $\mathbf{T} 7 \mathbf{B z}-\mathbf{T S O}_{2}$ and $1.60 \%$ for $\mathbf{T} 7 \mathbf{B z}-\mathbf{D p}_{2}$ - mainly due to a 


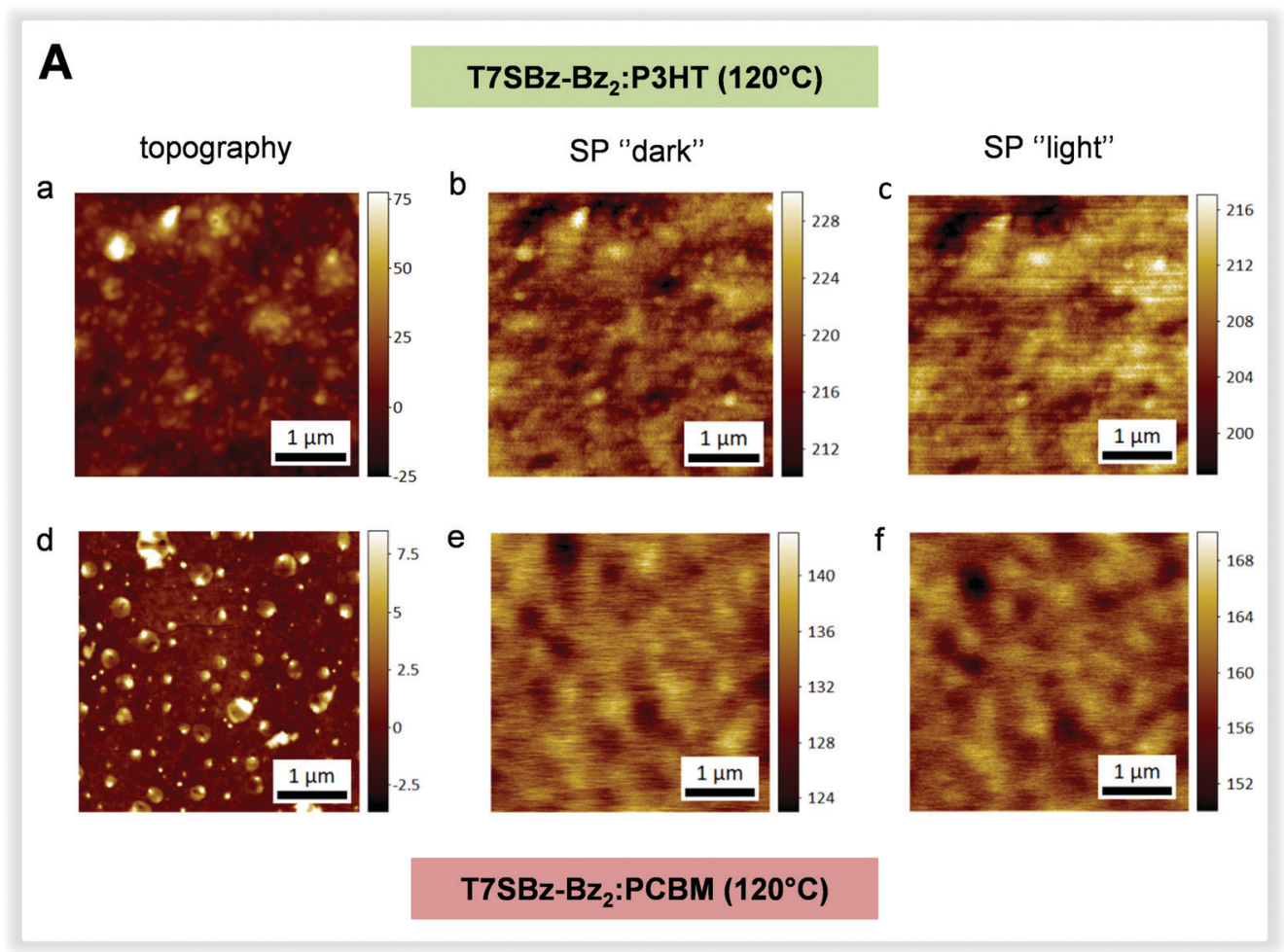

B

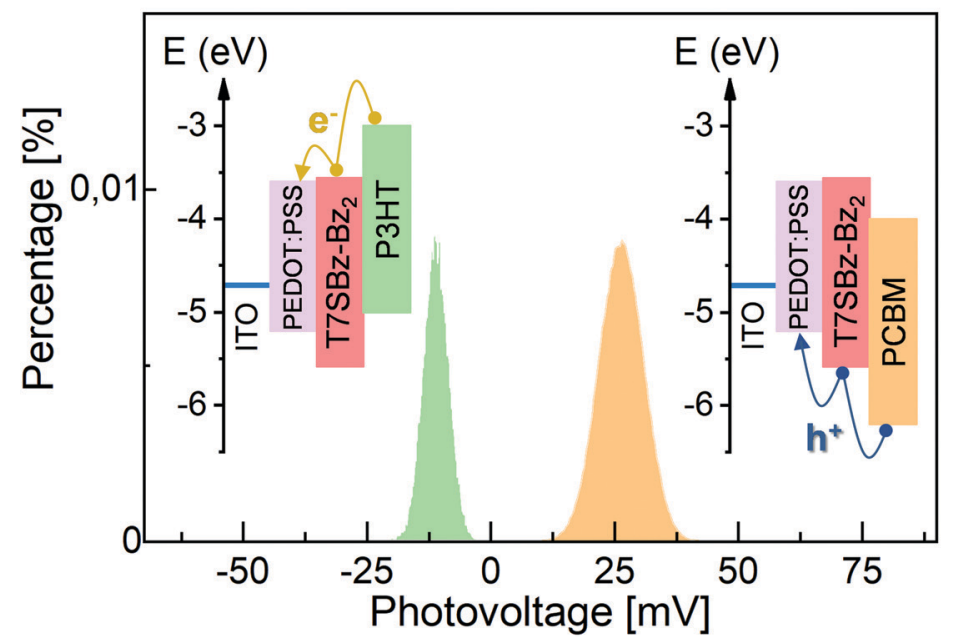

Fig. 4 (A) Topography ( $a$ and d) and surface potential (b, c dark and e, f under illumination) images of T7S-Bz-Bz $\mathbf{z}_{2}$ compound blended with P3HT (upper panel) and PCBM (lower panel). (B) Histogram distribution of the surface photovoltage (i.e., the difference between the SP values of figure Ac, Ab and Af, Ae). Inset: Schematic HOMO/LUMO energy level alignment showing the prevalence of electrons (holes) transport when the heptamer is blended with P3HT (PCBM).

lowering of the $J_{\mathrm{sc}}$ and $V_{\mathrm{oc}}$ values. Conversely, changing the $\mathrm{D}$ units with thioalkylated units as in $\mathbf{T} 7 \mathbf{S B z}-\mathbf{B z}_{2}$ results in a strong improvement in PCE efficiency, which reaches a value of $4.32 \%$. The presence of extra sulfur atoms in the side chains allows (i) to maintain suitable and reasonable $V_{\mathrm{oc}}$ value $(0.58 \mathrm{~V})$, while promoting and enhancing the photoabsorption step, and (ii) to remarkably increase the $J_{\mathrm{sc}}$ (up to $11.42 \mathrm{~mA} \mathrm{~cm}{ }^{2}$ ) and $\mathrm{FF}$ value (up to 0.65 ).

All blend samples were subjected to AFM, WAXS and grazing incidence WAXS (GIWAXS) measurements in order to correlate morphological investigations with structural data. As far as structural features are concerned, XRD patterns of the blends formed with $\mathbf{T} \mathbf{7} \mathbf{B z}-\mathbf{B z}_{2}$ and $\mathbf{T} \mathbf{7} \mathbf{S B z}-\mathbf{B z}_{2}$ and PCBM are featureless (Fig. S28, ESI $\dagger$ ), indicative of an amorphous state. In contrast, the XRD profiles of the blends of $\mathbf{T} 7 \mathbf{B z}-\mathbf{D p}_{2}$ and $\mathbf{T} \mathbf{7} \mathbf{B z}-\mathbf{T S O}_{2}$ with PCBM show sharp peaks - with periodicities ascribable to the same distances of the pristine films as reported in Fig. 1C indicating that the PCBM does not perturb the aggregation modality of the oligomers, which keep forming ordered structures in the blend. Notably, both WAXS and GIWAXS 
Table 2 Short-circuit current density $\left(J_{\mathrm{sc}}\right)$, open-circuit voltage $\left(V_{\text {oc }}\right)$, fill factor (FF), and power conversion efficiency (PCE) of tested BHJ solar cells (average values collected from five devices)

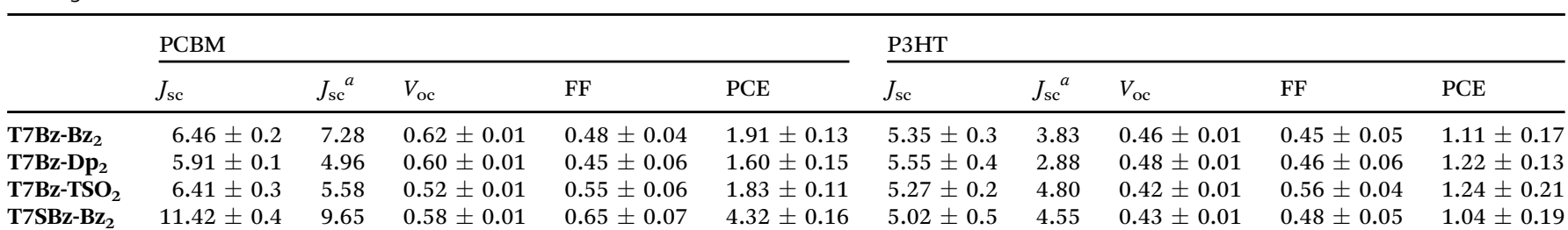

${ }^{a} J_{\mathrm{sc}}$ from EQE $\left(J_{\mathrm{sc}}\right.$ in $\mathrm{mA} \mathrm{cm}{ }^{-2} ; \mathrm{FF}$ and PCE in $\% ; V_{\mathrm{oc}}$ in $\left.\mathrm{V}.\right)$.

A

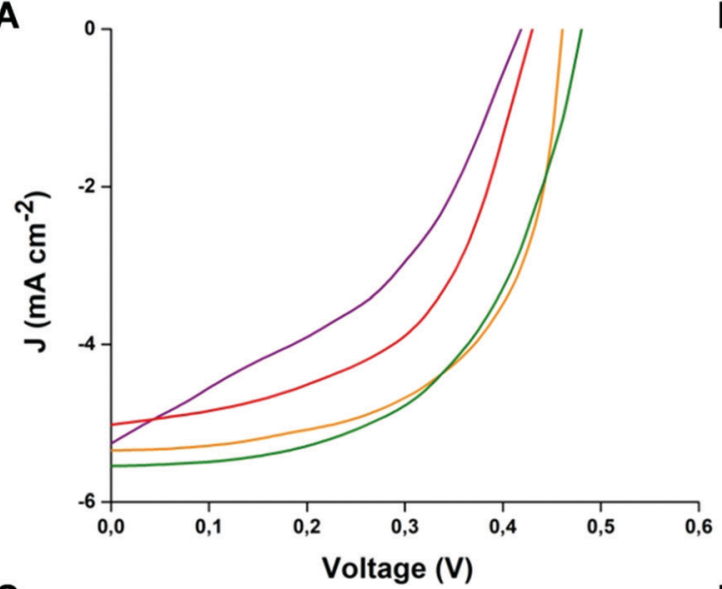

C

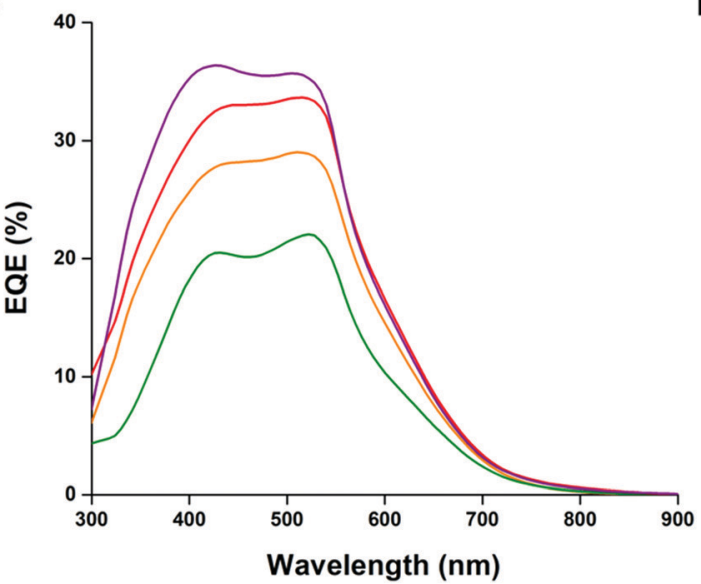

B

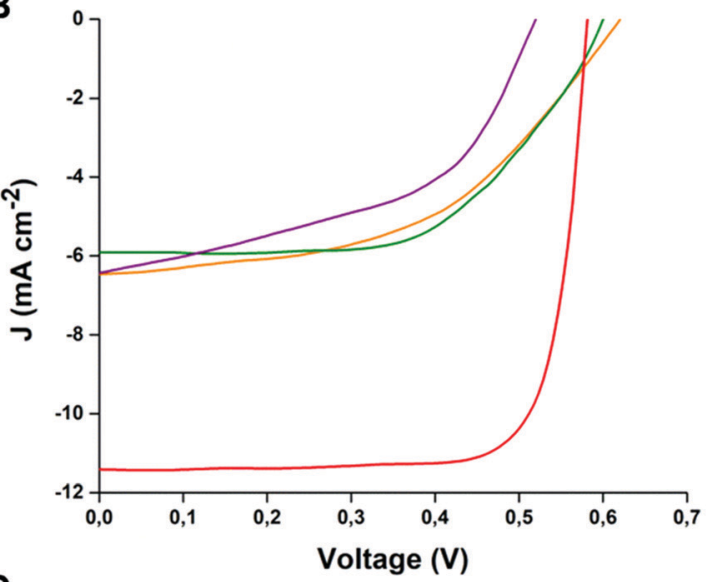

D

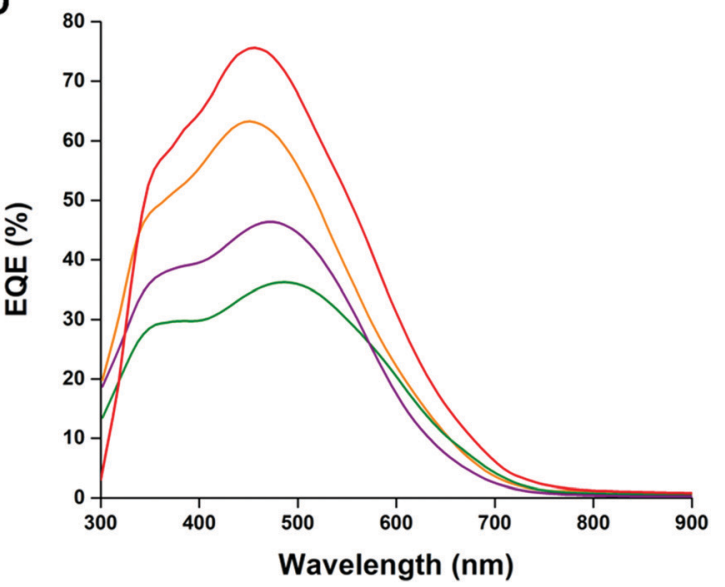

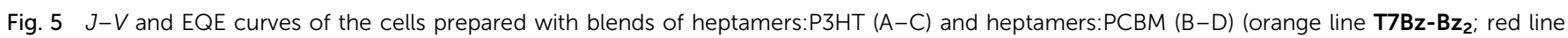

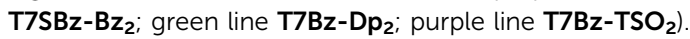

measurements, which allow to highlight parallel and perpendicular planes to the sample surface, respectively, give similar results. According to Scherrer analysis, crystalline domains in the range of $47-60 \mathrm{~nm}$ for T7Bz-Dp 2 and $20-26 \mathrm{~nm}$ for T7Bz-TSO ${ }_{2}$ are formed (Table S11, ESI $\dagger$ ). Further investigations of the morphology of the active layers were performed through AFM measurements (Fig. 6). All PCBM blended films exhibit similar thicknesses on the order of $\sim 200 \mathrm{~nm}$ (Table S9, ESI $\dagger$ ). However, those formed with $\mathbf{T} \mathbf{7} \mathbf{B z}-\mathbf{B z} \mathbf{z}_{\mathbf{2}}$ and $\mathbf{T} \mathbf{7} \mathbf{S B z}-\mathbf{B z}_{\mathbf{2}}$ appear to be more uniform and smoother (with root-mean-square (RMS) surface roughness values of $2 \mathrm{~nm}$ ) compared to those embedding $\mathbf{T} \mathbf{7} \mathbf{B z}-\mathbf{D} \mathbf{p}_{\mathbf{2}}$ and $\mathbf{T} \mathbf{7} \mathbf{B z}-\mathbf{T S O} \mathbf{S}_{\mathbf{2}}$, which are rather coarse
(RMS values of 4 and $16 \mathrm{~nm}$, respectively). AFM images show that the blend of $\mathbf{T} \mathbf{7} \mathbf{S B z}-\mathbf{B z}_{2}$ and PCBM presents round-shaped dark regions of $\sim 200-300 \mathrm{~nm}$ constituted by domains of

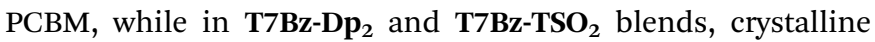
domains of the heptamers with needle-like shape are present in agreement with XRD measurements (Fig. 6A). The phase segregation observed in all the blends formed by the heptamers with PCBM is generally considered a valuable characteristic in $\mathrm{BHJ}$. Indeed, it can lead to a higher interfacial area between D and A domains, enhancing exciton dissociation. ${ }^{58,59}$ The measured BHJs performances suggest that the exclusive crystallinity of the donor material does not result in high PCE efficiency, 


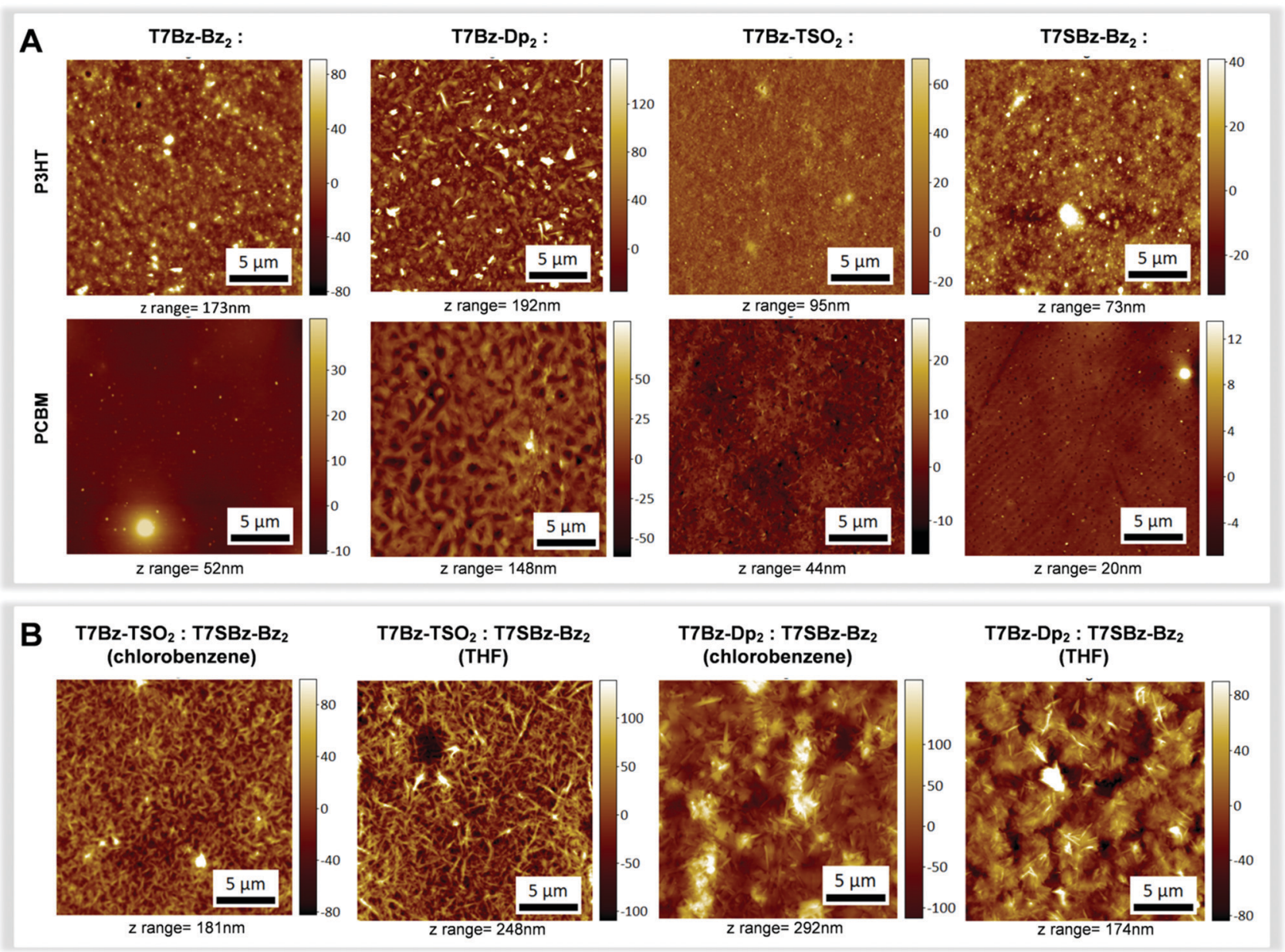

Fig. 6 (A) AFM images of the blends of heptamers:P3HT (top) and heptamers:PCBM (bottom). (B) AFM images of the blends $\mathrm{T7Bz}^{-\mathrm{TSO}} \mathbf{2}_{\mathbf{2}}: \mathrm{T7SBz}-\mathrm{Bz}_{\mathbf{2}}$ and

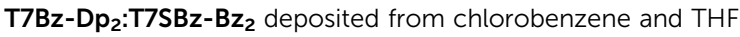

whereas the presence of nanoscopic PCBM aggregates may favourably affect the performances. This can be rationalized considering that PCBM aggregation can prevent charge recombination while promoting the generation of free, extractable charges. ${ }^{59,60}$

Heptamers:P3HT blends. Heptamers blended with P3HT act as acceptor material derivatives, thus confirming their ambivalent character in organic photovoltaic devices. However, PCE values are reduced compared to those measured when the heptamers are employed as D materials in blend with PCBM, being in the range of $1.04-1.24 \%$. In particular, the most affected parameter, which results in a reduction in the efficiency of the cells, is the $J_{\text {sc }}$ value. As shown in Table 2, changing the peripheral A groups or the D units of the heptamer has only a marginal effect on PCE efficiency. For all samples, the XRD patterns of the blends reveal the presence of a small peak at $5^{\circ}(1.7 \mathrm{~nm})$ attributable to P3HT crystalline domains (Fig. S28, ESI $\dagger$ ). ${ }^{61}$ The same behaviour observed in the PCBM blends is also seen for $\mathbf{T} 7 \mathbf{B z}-\mathbf{B z}_{2}$ and $\mathbf{T S 7} \mathbf{B z}-\mathbf{B z}_{2}$ P3HT blends, where the oligomer does not crystallize. From the XRD measurements, the average size of the coherent crystalline domains attributed to $\mathrm{P} 3 \mathrm{HT}$ is estimated, from the Scherrer equation, as $13 \mathrm{~nm}$ (Table S11, ESI $\dagger$ ), while the AFM morphology images, being more sensitive to the larger tail of the distribution of the crystalline domains, show the presence of spherically shaped clusters with sizes of tens of nanometers, with the largest ones being up to $\sim 100 \mathrm{~nm}$ (Fig. 6A). Accordingly, the previously described behaviour is also revealed for T7Bz-Dp ${ }_{2}$ and T7Bz-TSO $_{2}$ in blend with P3HT, with both heptamers presenting a strong tendency toward the formation of nano-crystalline domains (Fig. S28, ESI $\dagger$ ). This is also evident from the AFM topography images, where alongside the P3HT spherical clusters, we observe the presence of needle-like crystals also found in the case of PCBM blends. In particular, in the T7Bz-Dp $\mathbf{p}_{2}$ compound, the heptamers crystals can reach sizes up to hundreds of nanometers. Furthermore, AFM measurements show that all the heptamer:P3HT blend films, deposited with the same technique and in the same conditions as those embedding PCBM, present a smaller thickness $(\sim 100 \mathrm{~nm})$ and a higher $R_{\mathrm{RMS}}$ roughness $(10-32 \mathrm{~nm})$ (Table S9, ESI $\dagger)$.

The external quantum efficiencies (EQE) of all cells (Fig. 5C and D) were also used for calculation of $J_{\text {sc }}$, obtaining data consistent with the experimental findings (see Table 2), thus 
Table 3 Short-circuit current density $\left(J_{s c}\right)$, open-circuit voltage $\left(V_{o c}\right)$, fill factor (FF) and power conversion efficiency (PCE) of tested binary all-smallmolecule $\mathrm{BHJ}$ solar cells (average values collected from five devices)

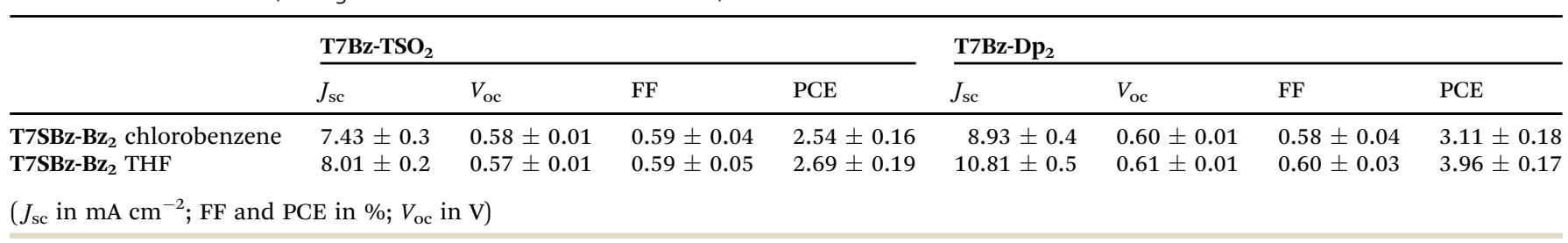

confirming the best performance of the devices prepared with $\mathbf{T} 7 \mathbf{S B z}-\mathbf{B z}_{2}$ and $\mathbf{T} 7 \mathbf{B z}-\mathbf{T S O}_{2}$, when blended with PCBM and P3HT, respectively. Additionally, the EQE profiles follow the trend observed in the UV-Vis spectra of heptamers in thin film (Fig. 3C and D), indicating that the whole absorption effectively contributes to the photocurrent.

Heptamer:heptamer blends. The bifunctional photoactive behaviour of the newly synthesized materials enabled to investigate binary NF-SM OSCs made by blending heptamers with different electron affinities. Although different combinations of heptamers blends are possible, the electrochemical LUMO level alignment was used as a guiding factor. Accordingly, heptamer T7 $\mathbf{S B z}^{-B_{2}} \mathbf{2}_{2}$ (LUMO eV -3.56) was used in a 1:1 weight ratio blend with either T7Bz-TSO ${ }_{2}$ (LUMO eV -3.63) or T7Bz-Dp (LUMO eV -3.45), working as A and D species, respectively. Additionally, thanks to the absence of fullerene derivatives and the optimal solubility and miscibility of the heptamers in a non-chlorinated solvent, the devices were also processed from THF, in addition to chlorobenzene as the formerly used solvent. The relative thin film absorption spectra are reported in Fig. S26 (ESI + ). In both $\mathbf{T} 7 \mathbf{S B z}-\mathbf{B z}_{2}: \mathbf{T}_{\mathbf{B}} \mathbf{B z}-\mathbf{T S O}_{\mathbf{2}}$ and $\mathbf{T} 7 \mathbf{S B z}-\mathbf{B z}_{2}$ : T7Bz-Dp $_{2}$ blends, the absorption spectra of thin films deposited from THF are red shifted of $\sim 20 \mathrm{~nm}$ compared to those deposited from chlorobenzene, hence evidencing a reduced optical band gap. The photovoltaic characteristics of the devices, having the previously adopted device configuration ITO/PEDOT:PSS/active layer/Al, are reported in Table 3 and the corresponding $J-V$ plots of the best-performing cells in Fig. S30 (ESI $\dagger$ ).

The bifunctionality of these push-pull thiophene-based small molecules, i.e., $\mathbf{T} 7 \mathbf{S B z}-\mathbf{B z}_{2}$, is further proved by obtaining interesting and promising PCE values ranging between 2.54$3.96 \%$. This is of particular regard, considering the potential use of a non-chlorinated and less harmful solvent such as THF. Fig. 6B reports the AFM of the blends, which present an anisotropic morphology independently from the solvent employed (i.e., THF or chlorobenzene). Diffraction profiles of the heptamer:heptamer blends indicate that the crystalline component is constituted by $\mathbf{T} \mathbf{7 B z}-\mathbf{D p}_{2}$ or $\mathbf{T} \mathbf{7} \mathbf{B z}-\mathbf{T S O}_{2}$ with a size in the range of $47-63 \mathrm{~nm}$ (Fig. S29 and Table S12, ESI $\dagger$ ). Note that the formation of crystalline domains is considered favorable in NF-SM OSCs as it avoids excessive intermixing between $\mathrm{D}$ and $\mathrm{A}$ materials thus overcoming the problems related to the charge recombination in blend films. ${ }^{62,63}$ Furthermore, thin films deposited from THF show a lower thickness compared to those deposited from chlorobenzene whose roughness is related to the dimension of the formed crystals (Table S10, ESI $\dagger$ ).

It is important to note that these couples of D:A heptamers present negligible values of $\Delta E(\mathrm{HOMO})_{\text {offset }}(0$ and $-0.01 \mathrm{eV}$ for T7SBz-Bz 2 :T7Bz-Dp $\mathbf{P}_{2}$ and $\mathbf{T} 7 \mathbf{S B z}-\mathbf{B z}_{2}: \mathbf{T} 7 \mathbf{B z}-\mathbf{T S O}_{2}$, respectively) and $\Delta E(\text { LUMO })_{\text {offset }}\left(0.11\right.$ and $0.07 \mathrm{eV}$ for T7SBz-Bz 2 :T7Bz-Dp $\mathbf{p}_{2}$

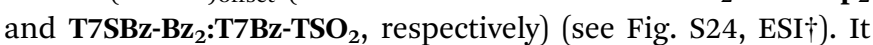
has recently been shown that having fullerene-free organic solar cells with a low HOMO and LUMO offset between D/A blend could be a tool to improve the OSC efficiencies. Indeed, it was found that the small driving force for charge separation resulting from these $\Delta E_{\text {offset }}$ close to zero allows ultrafast charge transfer and a suppression of the energy losses that hinder the device performances. ${ }^{41-43}$

Finally, the $V_{\text {oc }}$ values measured for heptamer:heptamer blends result to be lower than expected from CV/optical measurements. This suggests that further optimization of the active layer can potentially lead to better performance. ${ }^{64,65}$ In this regard, we expect that higher efficiencies can be achieved through the optimization of processing conditions - e.g., by varying D/A weight ratio, selecting the solvent or mixed solvent, adding solid additives, changing deposition techniques - as well as employing multiple post-treatment methods, including vacuum drying, solvent annealing, and thermal annealing. ${ }^{64-67}$ Furthermore, as recently reported, optimizing the device architecture through the introduction of organic electron transport layers (ETLS) could be a goal to realize highly efficient NF-SM OSCs. ${ }^{68}$

\section{Conclusions}

In this study, we synthesized and characterized a new family of thiophene-based small molecules with a $\mathrm{D}-\mathrm{A}^{\prime}-\mathrm{D}-\mathrm{A}-\mathrm{D}-\mathrm{A}^{\prime}-\mathrm{D}$ sequence. An extensive study of the influence of the different substitution pattern - induced either by introducing an extra sulfur atom in the side chains or by varying the strength of the acceptor unit (benzothiadiazole $v s$. diketopirrole $v s$. thiophene$S, S$-dioxide) - on the variation of the chemical-physical properties is reported. In addition, a multiple approach including $\mathrm{X}$-ray diffraction, thermal analysis and DFT calculations has been employed to investigate the aggregation features of the oligomers. A greater tendency to form ordered arrays was observed when non-covalent intra- and intermolecular interactions, such as $\mathrm{S} \cdots \mathrm{S}$, or $\mathrm{S} \cdots \mathrm{O}$, are present in the molecule. Cyclic voltammetry combined with KPFM measurements allowed to identify that these 
heptamers present a bifunctional photoactive behaviour, as they can be employed either as donor or acceptor materials when blended with PCBM or P3HT, respectively. Furthermore, we demonstrated that thanks to their bifunctional photoactivity, binary NF-SM OSCs can be prepared by blending distinct heptamers with different electron affinities. In this case, PCE efficiency of $2.54-3.96 \%$ can be achieved despite very low HOMO and LUMO offsets which nevertheless guarantee the operation of the device. Our work highlights that further exploration of novel pairs of donor and acceptor thiophene based small molecules from the point of view of their energy levels, morphology, and surface potential (SP) distribution can be useful to provide guidelines for the design of future multifunctional materials and increase the performances of NF-SM OSCs devices.

\section{Author contributions}

FM conducted DFT analysis and analyzed the data. AC and AB carried out AFM and KPFM measurements. MG made XRD measurements and analyzed the data. MZ and FDM prepared and characterized the heptamers. AZ carried out CV experiments. ES made thermal analysis. MM, ML prepared and characterized $\mathrm{BHJ}$ solar cells. FP performed EQE analysis. MZ contributed to the writing of the paper. FDM coordinated the project and wrote the paper with inputs from the other authors. All authors have approved the final version of the manuscript.

\section{Conflicts of interest}

There are no conflicts to declare.

\section{Acknowledgements}

FDM and MZ acknowledge the UE project INFUSION (Engineering optoelectronic INterfaces: a global action intersecting FUndamental conceptS and technology implementatION of self-organized organic materials, Proposal number: 734834). FP thanks the National Centre for Research and Development (NCBiR) for the financial support via the LIDER program (NCBiR grant no. LIDER/ 28/0067/L-7/15/NCBR/2016). This work was partially supported by the Italian MIUR (PRIN 20173L7W8K).

\section{Notes and references}

1 K. A. Mazzio and C. K. Luscombe, Chem. Soc. Rev., 2015, 44, 78.

2 A. Wadsworth, Z. Hamid, J. Kosco, N. Gasparini and I. McCulloch, Adv. Mater., 2020, 32, 2001763.

3 W. Liu, X. Xu, J. Yuan, M. Leclerc, Y. Zou and Y. Li, ACS Energy Lett., 2021, 6, 598.

4 P. Cheng and Y. Yang, Acc. Chem. Res., 2020, 53, 218.

5 Y. Che, Y. Zhang, Y. Yang, C.-H. Liu, R. Izquierdo, S. S. Xiao and D. F. Perepichka, J. Org. Chem., 2020, 85, 52.

6 Y. Zhou, Y. Qin, C. Ni and Y. Xie, J. Appl. Polym. Sci., 2020, 137, e49006.
7 E. Salatelli, M. Marinelli, M. Lanzi, S. Dell'Elce, A. Liscio, M. Gazzano and F. Di Maria, J. Phys. Chem. C, 2018, 122, 4156.

8 Q. Liu, Y. Jiang, K. Jin, J. Qin, J. Xu, W. Li, J. Xiong, J. Liu, Z. Xiao, K. Sun, S. Yang, X. Zhang and L. Din, Sci. Bull., 2020, 65, 272.

9 L. Zhan, S. Li, X. Xia, Y. Li, X. Lu, L. Zuo, M. Shi and H. Chen, Adv. Mater., 2021, 33, 2007231.

10 R. Zhou, Z. Jiang, C. Yang, J. Yu, M. A. Adil, D. Deng, W. Zou, J. Zhang, K. Lu, W. Ma, F. Gao and Z. Wei, Nat. Commun., 2019, 10, 5393.

11 B. Yadagiri, K. Narayanaswamy, S. Revoju, B. Eliasson, G. D. Sharma and S. P. Singh, J. Mater. Chem. C, 2019, 7, 709.

12 H. Tran, M. Haris, T. Ahn and S. K. Lee, Mol. Cryst. Liq. Cryst., 2020, 705, 35.

13 B. Kan, Y. Kan, L. Zuo, X. Shi and K. Gao, InfoMat, 2021, 3, 175.

14 X. Wan, C. Li, M. Zhang and Y. Chen, Chem. Soc. Rev., 2020, 49, 2828.

15 K. He, P. Kumar, Y. Yuan and Y. Li, Mater. Adv., 2021, 2, 115.

16 A. Armin, W. Li, O. J. Sandberg, Z. Xiao, L. Ding, J. Nelson, D. Neher, K. Vandewal, S. Shoaee, T. Wang, H. Ade, T. Heumuller, C. Brabec and P. Meredith, Adv. Energy Mater., 2021, 11, 20003570.

17 M. E. Kose, J. Phys. Chem. A, 2012, 116, 12503.

18 J. Du, M. C. Biewer and M. C. Stefan, J. Mater. Chem. A, 2016, 4, 15771.

19 H. Cao, N. Bauer, C. Pang, J. Rech, W. You and P. A. Rupar, ACS Appl. Energy Mater., 2018, 1, 7146.

20 C. Zhao, F. Yang, D. Xia, Z. Zhang, Y. Zhang, N. Yan, S. You and W. Li, Chem. Commun., 2020, 56, 10394.

21 S. UrRehman, A. Alam, S. Sadaf, S. R. Khan, M. Shoaib, A. Q. Khan, M. Khan and W. UrRehman, J. Mol. Model., 2020, 26, 154.

22 D. Gedewaf, X. Pan and M. R. Andersson, Front. Mater., 2020, 7, 280.

23 Z. Wang, K. Gao, Y. Kan, M. Zhang, C. Qiu, L. Zhu, Z. Zhao, X. Peng, W. Feng, Z. Qian, X. Gu, A. K.-Y. Jen, B. Z. Tang, Y. Cao, Y. Zhang and F. Liu, Nat. Commun., 2021, 12, 332.

24 B. Y. Antwi, R. G. D. Taylor, J. Cameron, R. B. Owoare, R. Kingsford-Adaboh and P. J. Skabara, RSC Adv., 2016, 6, 98797.

25 Z. Wang, L. Zhu, Z. Shuai and Z. Wei, Macromol. Rapid Commun., 2017, 38, 1700470.

26 P. Gautam, R. Sharma, R. Misra, M. L. Keshtov, S. A. Kuklin and G. D. Sharma, Chem. Sci., 2017, 8, 2017.

27 Z. Zhang, J. Yuan, Q. Wei and Y. Zou, Front. Chem., 2018, 6, 414.

28 S. Alam, M. S. Akhtar, Abdullah, E.-B. Kim, H.-S. Shin and S. Ameen, Appl. Sci., 2021, 11, 646.

29 J. Hou, O. Inganas, R. H. Friend and F. Gao, Nat. Mater., 2018, 17, 119.

30 A. Karki, A. J. Gillett, R. H. Friend and T.-Q. Nguyen, Adv. Energy Mater., 2020, 11, 2003441. 
31 B. C. Schroeder, R. S. Ashraf and I. McCulloch, ThiopheneBased High-Performance Donor Polymers for Organic Solar Cells, Wiley-VCH Verlag GmbH \& Co. KGaA, 2014.

32 C. Duan, K. Gao, F. J. M. Colberts, S. C. J. Meskers, M. M. Wienk and R. A. J. Janssen, Adv. Energy Mater., 2017, 7, 1700519.

33 T. Xu, Y. Chang, C. Yan, Q. Yang, Z. Kan, R. Singh, M. Kumar, G. Li, S. Lu and T. Duan, Sustainable Energy Fuels, 2020, 4, 2680.

34 J. Liu, H. Lu, Y. Liu, J. Zhang, C. Li, X. Zu and Z. Bo, ACS Appl. Mater. Interfaces, 2020, 12, 10746.

35 J. Pan, L. Wang, W. Chen, S. Sang, H. Sun, B. Wu, X.-C. Hang, Z. Sun and W. Huang, J. Mater. Chem. C, 2020, 8, 6749.

36 M. Marinelli, M. Lanzi, A. Liscio, A. Zanelli, M. Zangoli, F. Di Maria and E. Salatelli, J. Mater. Chem. C, 2020, 8, 4124.

37 F. Di Maria, M. Biasiucci, F. P. Di Nicola, E. Fabiano, A. Zanelli, M. Gazzano, E. Salatelli, M. Lanzi, F. Della Sala, G. Gigli and G. Barbarella, J. Phys. Chem. C, 2015, 119, 27200.

38 J. Song, X. Xue, B. Fan, L. Huo and Y. Sun, Mater. Chem. Front., 2018, 2, 1626.

39 G. Qiu, Z. Jiang, Z. Ni, H. Wang, H. Dong, J. Zhang, X. Zhang, Z. Shu, K. Lu, Y. Zhen, Z. Wei and W. Hu, J. Mater. Chem. C, 2017, 5, 566.

40 H. Shi, W. Fu, M. Shi, J. Ling and H. Chen, J. Mater. Chem. A, 2015, 3, 1902.

41 J. Liu, S. Chen, D. Qian, B. Gautam, G. Yang, J. Zhao, J. Bergqvist, F. Zhang, W. Ma, H. Ade, O. Inganäs, K. Gundogdu, F. Gao and H. Yan, Nat. Energy, 2016, $1,1$.

42 H. Fu, Y. Wang, D. Meng, Z. Ma, Y. Li, F. Gao, Z. Wang and Y. Sun, ACS Energy Lett., 2018, 3, 2729.

43 S. Li, L. Zhan, C. Sun, H. Zhu, G. Zhou, W. Yang, M. Shi, C.-Z. Li, J. Hou, Y. Li and H. Chen, J. Am. Chem. Soc., 2019, 141, 3073.

44 M. Zangoli, M. Gazzano, F. Monti, L. Maini, D. Gentili, A. Liscio, A. Zanelli, E. Salatelli, G. Gigli, M. Baroncini and F. Di Maria, ACS Appl. Mater. Interfaces, 2019, 11, 16864.

45 F. Di Maria, M. Zangoli, I. Palamá, E. Fabiano, A. Zanelli, M. Monari, A. Perinot, M. Caironi, V. Maiorano, A. Maggiore, M. Pugliese, E. Salatelli, G. Gigli, I. Viola and G. Barbarella, Adv. Funct. Mater., 2016, 26, 6970.

46 F. Di Maria and G. Barbarella, J. Sulfur Chem., 2013, 34, 627. 47 M. Melucci, L. Favaretto, C. Bettini, M. Gazzano, N. Camaioni, P. Maccagnani, P. Ostoja, M. Monari and G. Barbarella, Chem. - Eur. J., 2007, 13, 10007.

48 M. B. Ros, J. L. Serrano, M. R. de la Fuente and C. L. Folcia, J. Mater. Chem., 2005, 15, 5093.

49 P.-S. Lin, Y. Shoji, S. N. Afraj, M. Ueda, C.-H. Lin, S. Inagaki, T. Endo, S.-H. Tung, M.-C. Chen, C.-L. Liu and
T. Higashihara, ACS Appl. Mater. Interfaces, 2021, 13(27), 31898-31909.

50 C. Zhao, F. Yang, D. Xia, Z. Zhang, Y. Zhang, N. Yan, S. You and W. Li, Chem. Commun., 2020, 56, 10394.

51 X. Zhang, Z. Gong, J. Li and T. Lu, Intermolecular SulfurOxygen, J. Chem. Inf. Model., 2015, 55, 2138.

52 S. Canola, L. Mardegan, G. Bergamini, M. Villa, A. Acocella, M. Zangoli, L. Ravotto, S. A. Vinogradov, F. Di Maria, P. Ceroni and F. Negri, Photochem. Photobiol. Sci., 2019, 18, 2180.

53 S. Ellinger, K. R. Graham, P. Shi, R. T. Farley, T. T. Steckler, R. N. Brookins, P. Taranekar, J. Mei, L. A. Padilha, T. R. Ensley, H. Hu, S. Webster, D. J. Hagan, E. W. Van Stryland, K. S. Schanze and J. R. Reynolds, Chem. Mater., 2011, 23, 3805.

54 R. Valero, R. Costa, I. de P. R. Moreira, D. G. Truhlar and F. Illas, J. Chem. Phys., 2008, 128, 114103.

55 W. Melitz, J. Shen, A. C. Kummel and S. Lee, Surf. Sci. Rep., 2011, 66, 1.

56 V. Palermo, M. Palma and P. Samorì, Adv. Mater., 2006, 18, 145.

57 V. Palermo, M. B. J. Otten, A. Liscio, E. Schwartz, P. A. J. de Witte, M. A. Castriciano, M. M. Wienk, F. Nolde, G. De Luca, J. J. L. M. Cornelissen, R. A. J. Janssen, K. Müllen, A. E. Rowan, R. J. M. Nolte and P. Samorì, J. Am. Chem. Soc., 2008, 130, 14605.

58 C. Zhong, J. A. Bartelt, M. D. McGehee, D. Cao, F. Huang, Y. Cao and A. J. Heeger, J. Phys. Chem. C, 2015, 119, 26889.

59 H.-C. Liao, C.-C. Ho, C.-Y. Chang, M.-H. Jao, S. B. Darling and W.-F. Su, Mater. Today, 2013, 16, 326336.

60 T. Agostinelli, S. Lilliu, J. G. Labram, M. Campoy-Quiles, M. Hampton, E. Pires, J. Rawle, O. Bikondoa, D. D. C. Bradley, T. D. Anthopoulos, J. Nelson and J. E. Macdonald, Adv. Funct. Mater., 2011, 21, 1701.

61 W. Ma, C. Yang, X. Gong, K. Lee and A. J. Heeger, Adv. Funct. Mater., 2005, 15, 1617.

62 J. Zhang, H. S. Tan, X. Guo, A. Facchetti and H. Yan, Nat. Energy, 2018, 3, 720.

63 Q. Zhang, Z. Chen, W. Ma, Z. Xie and Y. Han, J. Mater. Chem. C, 2019, 7, 12560.

64 Y. Xie, W. Zhou, J. Yin, X. Hu, L. Zhang, X. Meng, Q. Ai and Y. Chen, J. Mater. Chem. A, 2016, 4, 6158.

65 Z. Zhang, Q. Wu, D. Deng, S. Wu, R. Sun, J. Min, J. Zhang and Z. Wei, J. Mater. Chem. C, 2020, 8, 15385.

66 G. Qin, L. Zhang, D. Yuan, H. Jiang, W. Tang, M. Chen, W. Wu, Y. Cao and J. Chen, J. Mater. Chem. A, 2019, 7, 25978.

67 J. Min, O. K. Kwon, C. Cui, J.-H. Park, Y. Wu, S. Y. Park, Y. Li and C. J. Brabec, J. Mater. Chem. A, 2016, 4, 14234.

68 R. Sorrentino, E. Kozma, S. Luzzati and R. Po, Energy Environ. Sci., 2021, 14, 180. 